\title{
The Effect of Surface Electrical Stimulation on Swallowing in Dysphagic Parkinson Patients
}

\author{
Laura W. J. Baijens · Renée Speyer • \\ Valeria Lima Passos • Walmari Pilz • \\ Nel Roodenburg • Père Clavé
}

Received: 18 July 2011/Accepted: 14 December 2011/Published online: 12 January 2012

(C) The Author(s) 2012. This article is published with open access at Springerlink.com

\begin{abstract}
Surface electrical stimulation has been applied on a large scale to treat oropharyngeal dysphagia. Patients suffering from oropharyngeal dysphagia in the presence of Parkinson's disease have been treated with surface electrical stimulation. Because of controversial reports on this treatment, a pilot study was set up. This study describes the effects of a single session of surface electrical stimulation using different electrode positions in ten patients with idiopathic Parkinson's disease (median Hoehn and Yahr score: II) and oropharyngeal dysphagia compared to ten age- and gender-matched healthy control subjects during videofluoroscopy of swallowing. Three different electrode positions were applied in random order per subject. For each electrode position, the electrical current was respectively turned "on" and "off" in random order. Temporal, spatial, and visuoperceptual variables were scored by
\end{abstract}

L. W. J. Baijens $(\bowtie) \cdot$ W. Pilz

Department of Otorhinolaryngology, Head and Neck Surgery,

Maastricht University Medical Center, PO Box 5800,

6202 AZ Maastricht, The Netherlands

e-mail: laura.baijens@mumc.nl

\section{R. Speyer}

Institute of Health Studies, HAN University of Applied Sciences, Nijmegen, The Netherlands

\section{L. Passos}

Department of Methodology and Statistics, Maastricht

University, Maastricht, The Netherlands

\section{N. Roodenburg}

Department of Neurology, Maastricht University Medical

Center, Maastricht, The Netherlands

P. Clavé

Department of Surgery, Hospital de Mataró and CIBERehd, Instituto de Salud Carlos III, Mataró, Spain experienced raters who were blinded to the group, electrode position, and status (on/off) of the electrical current. Interrater and interrater reliabilities were calculated. Only a few significant effects of a single session of surface electrical stimulation using different electrode positions in dysphagic Parkinson patients could be observed in this study. Furthermore, significant results for temporal and spatial variables were found regardless of the status of the electrical current in both groups suggesting placebo effects. Following adjustment for electrical current status as well as electrode positions (both not significant, $P>0.05$ ) in the statistical model, significant group differences between Parkinson patients and healthy control subjects emerged. Further studies are necessary to evaluate the potential therapeutic effect and mechanism of electrical stimulation in dysphagic patients with Parkinson's disease.

Keywords Dysphagia - Deglutition disorders ·

Parkinson's disease - Electrical stimulation - Deglutition

The number of reports on the effects of therapies for dysphagia in Parkinson's disease is still small. Reviewing the literature, positive group tendencies may be observed, although no general conclusions can be drawn from these reports [1]. Surface electrical stimulation in dysphagic Parkinson patients has not been investigated in scientific studies before. Carnaby-Mann and Crary [2] describe a small but significant summary effect size for transcutaneous neuromuscular electrical stimulation (NMES) for dysphagia in a meta-analysis. The immediate effect of surface electrical stimulation on hyolaryngeal movement in normal individuals during swallowing is described by Humbert et al. [3], who observed a reduced hyolaryngeal elevation during electrical stimulation. They hypothesized 
a higher risk of further decreased hyolaryngeal elevation during electrical stimulation in dysphagic patients who are already suffering from reduced hyolaryngeal elevation. Ludlow et al. [4] observed that aspiration and pooling are significantly reduced in chronically dysphagic patients during surface electrical stimulation with low sensory threshold levels of stimulation. Almost all subjects showed depression of the hyoid bone during motor-level stimulation at rest. However, at the maximum motor level of stimulation during swallowing, no group change in aspiration was noted. In a systematic review, Clark et al. [5] reported promising findings of NMES, emphasizing the need for high-quality controlled trials in the future. Despite the fact that controversial reports have been published [3, 4], surface electrical stimulation is being applied on a large scale for oropharyngeal dysphagia due to diverse neurological etiologies, including Parkinson's disease. The application of surface electrical stimulation in dysphagic patients with Parkinson's disease without existing evidence of therapy effects in the literature has been the rationale for this present study. This study was performed to determine the immediate effect of a single session of surface electrical stimulation using different electrode positions and electrical current states in patients with Parkinson's disease and oropharyngeal dysphagia compared to the same protocol in a group of age- and gender-matched healthy control subjects during a videofluoroscopic swallowing study. The maximum tolerated motor level of stimulation was applied to all subjects during each swallowing act.

\section{Methods}

\section{Patients and Healthy Control Subjects}

Patients with a diagnosis of idiopathic Parkinson's disease and dysphagic complaints were recruited from several neurological departments from diverse hospitals all over the Netherlands. The dysphagic complaints ranged from mild to severe, including slow eating due to a reduced rate of spontaneous swallowing and to rigidity, hypokinesia, and bradykinesia of the masticatory, oral, or pharyngeal musculature involved in swallowing; oral or pharyngeal passage disorder; choking on food; and coughing while drinking or eating. The patients continued using their unaltered type and dose of antiparkinsonian medication for at least 2 months and their disease was stable at the time of inclusion. The Hoehn and Yahr (H\&Y) disability score was used as a clinical rating scale to assess the severity of the Parkinson's disease (Table 1) [6]. For each patient, an ageand gender-matched control was recruited. The following exclusion criteria were used for the patients as well as for the healthy control subjects: the presence of any other
Table 1 Demographics of dysphagic patients with Parkinson's disease and of healthy control subjects

\begin{tabular}{|c|c|c|c|c|c|}
\hline \multirow{2}{*}{$\begin{array}{l}\text { No. of } \\
\text { matched } \\
\text { pair of } \\
\text { subjects }\end{array}$} & \multirow[t]{2}{*}{ Sex } & \multirow{2}{*}{$\begin{array}{l}\text { Electrode } \\
\text { positions }\end{array}$} & \multicolumn{2}{|c|}{ Parkinson's disease patients } & \multirow{2}{*}{$\begin{array}{l}\text { Healthy } \\
\text { controls } \\
\text { Age } \\
\text { (years) }\end{array}$} \\
\hline & & & $\begin{array}{l}\mathrm{H} \& \mathrm{Y} \\
\text { scale }^{\mathrm{a}}\end{array}$ & $\begin{array}{l}\text { Age } \\
\text { (years) }\end{array}$ & \\
\hline 1 & M & I-II-III & I & 70 & 68 \\
\hline 2 & $\mathrm{~F}$ & III-I-II & II & 64 & 63 \\
\hline 3 & M & II-I-III & II & 50 & 46 \\
\hline 4 & M & III-II-I & III & 80 & 81 \\
\hline 5 & $\mathrm{~F}$ & III-I-II & II & 73 & 74 \\
\hline 6 & M & II-I-III & III & 57 & 53 \\
\hline 7 & $\mathrm{~F}$ & III-II-I & III & 62 & 60 \\
\hline 8 & M & I-II-III & II & 70 & 67 \\
\hline 9 & M & III-I-II & III & 70 & 70 \\
\hline 10 & $\mathrm{M}$ & II-I-III & I & 66 & 68 \\
\hline
\end{tabular}

${ }^{\mathrm{a}} \mathrm{H} \& \mathrm{Y}$ scale $=$ Hoehn and Yahr scale: the range of scores is I-V, where I indicates unilateral involvement, usually with minimal or no functional disability, and a V indicates confinement to bed or wheelchair unless aided [6]

neurological disease; inability to perform a swallow; deep brain stimulation (DBS); a Mini-Mental State Examination (MMSE) [7] score below 23; severe dyskinesia of head and neck (resulting in problems with VFS recording); mental depression; head and neck cancer; severe cardiopulmonary diseases; speech therapy during the past 6 months; and surgery on the swallowing mechanism or the central nervous system. Written informed consent was obtained from all patients and healthy control subjects. This study protocol was approved by the medical ethical committee of the university medical center.

\section{Protocol for Procedure of Surface Electrical} Stimulation

A two-channel battery-powered electrical stimulator (VitalStim ${ }^{\circledR}$ Therapy; frequency $80 \mathrm{~Hz}$, pulse width $700 \mu$ s, Chattanooga Group, Chattanooga, TN, USA) was used by an experienced laryngologist. The intensity of the bipolar electrode stimulation ranged from 0 to $25 \mathrm{~mA}$ for each channel. All subjects were familiarized with the application of the electrical stimulator before the experiment. All patients and healthy control subjects performed 12 swallows, each containing $10 \mathrm{cc}$ thin liquid barium boluses (low-density barium $40 \% \mathrm{w} / \mathrm{v}$ ). The following single session protocol of electrical stimulation during a standardized videofluoroscopic swallowing examination was performed using three different electrode positions applied in random order per subject. After the skin had been cleaned and shaven, electrodes $\left(V_{i t a l S t i m}{ }^{\circledR}\right.$, reference 59035) were placed on either side of the midline of the neck. In case of 

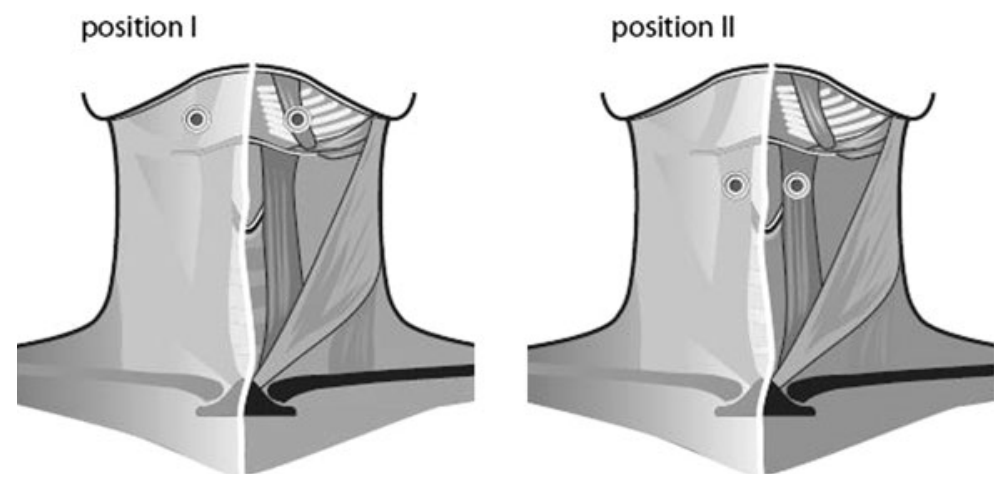

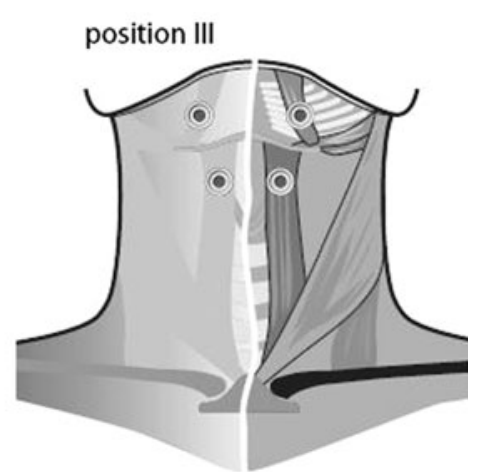

Fig. 1 Schematic illustration of the electrode positions. Position I = two electrodes horizontally above the hyoid bone (submental region); position $\mathrm{II}=$ two electrodes horizontally below the hyoid bone; position III = combination of positions I and II, with four electrodes connected on each side of the midline of the neck

preparatory cue. During the VFS, subjects were seated upright in lateral position wearing their dental prosthesis if present. The field of the videofluoroscopic image included the lips, oral cavity, cervical spine, and proximal cervical esophagus. A coin of 5 Euro cents was affixed to the retroauricular skin as a reference distance to correct for magnification (calibration). Videofluoroscopic images were obtained with a Philips Diagnost 97 system (Philips Medical Systems, Eindhoven, the Netherlands) and recorded on cassette at 25 frames per second using a mini-DV camerarecorder Panasonic AG-DVC30 (Matsushita Electric Industrial Co., Osaka, Japan). Sounds were recorded simultaneously. In the event of mild aspiration during a trial, the examination was continued. If severe aspiration was observed, the examination was ended. All VFS examinations were performed within 90-120 min after the intake of antiparkinsonian medication. During this period called the "on" motor phase, the levodopa motor effect is maximal [8].

\section{Selection of Variables}

For quantitative assessment of the swallow, specialized software was used (Image \& Physiology SL, Barcelona, Spain) to capture, digitize, and measure all swallowing sequences [9]. The capture rate was 25 frames per second. Timed variables were determined for the biomechanical analysis of swallowing [10]. The pharyngeal inlets and outlets were identified frame by frame by both raters during each pharyngeal swallow: moment of opening and closing of the glossopalatal junction (GPJ); moment of opening and closing of the velopharyngeal junction (VPJ); moment of opening and closing of the laryngeal vestibule (LV); and moment of opening and closing of the upper esophageal sphincter (UES). Using definitions of penetration and aspiration according to Rosenbek et al. [11], the frame exhibiting penetration or aspiration was marked as a timed event. 
Movement patterns of the hyoid bone were used as spatial variables to analyze the swallowing function quantitatively [12]. For each swallow, three reference points were marked in each video frame: the anterior/ superior corner of the hyoid bone and the anterior/inferior corner of the third and fifth cervical vertebral bodies. The $y$ axis was defined by the anterior/inferior corner of the third and fifth cervical vertebral bodies. Perpendicular to the $y$ axis, the $x$ axis crosses at the anterior/inferior corner of the third vertebral body. By marking these reference points in each frame, movements of the subject in any plane could be corrected (Fig. 2). Next, the extent of movement of the hyoid bone in the $x-y$ coordinate system over time was analyzed by means of specialized software.

Furthermore, visuoperceptual variables were scored for each VFS swallow. The variables are as follows: preswallow anterior and preswallow posterior spill, lingual pumping, swallow hesitancy, piecemeal deglutition, delayed initiation of the pharyngeal reflex, postswallow oral residue, postswallow vallecular pooling, postswallow pyriform sinus pooling, and the penetration aspiration scale of Rosenbek et al. [11, 13, 14]. In this 8-point scale (1-8), lower scores refer to normal functioning and higher scores refer to more severe disability. Following consensus training, two experts assessed each swallow independently at varying speed, ranging from normal to slow motion to frame-by-frame viewing. The consensus training in visuoperceptual evaluation was accompanied by a manual, including strict, well-defined guidelines to rate these ordinal variables. The exact interpretation per level of each of the three- or five-point scales was trained during five separate sessions with intervals of 1 week. During these intervals the expert raters had to accomplish test trials separately that were discussed during the next session.

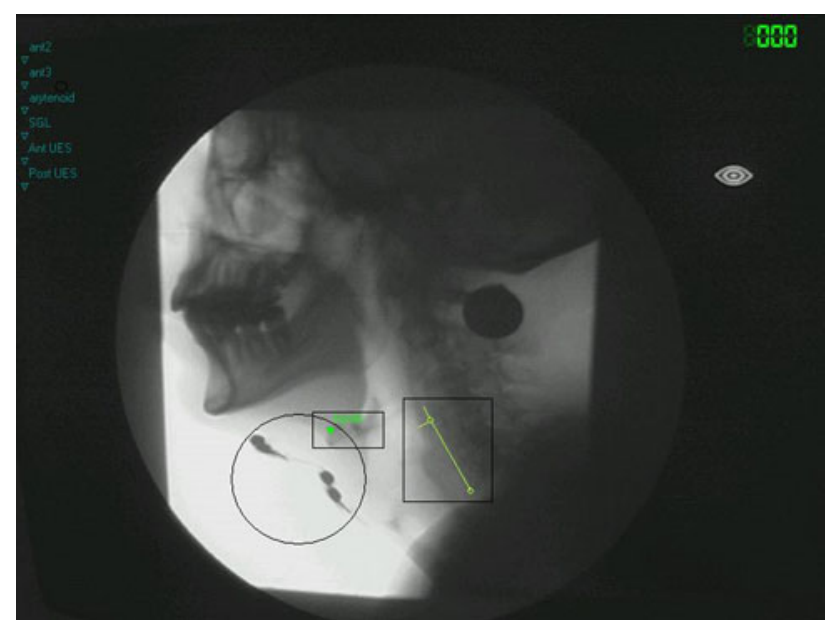

Fig. 2 Single frame of the videofluoroscopic recording showing the landmarks used for spatial measurements. The circle encloses four electrodes in position, the small rectangle includes the marked hyoid bone, and the large rectangle indicates the $x-y$ coordinate system
Previous research by Baijens et al. [15] has described the reliability or intra- and interrater agreement on several quantitative and qualitative parameters in VFS. Only parameters that proved to be sufficiently reliable have been included in the present study (Table 2). All measurements and ratings were performed independently by two experienced judges. Both judges were blinded to the group, electrode position, and status of the electrical current (on/off). To optimize blinding to the electrode position during VFS, four electrodes were placed on the skin according to the electrode positions mentioned above. Although the cables were connected to all four electrodes, only the electrodes of the position being studied during a specific swallowing act were stimulated (Fig. 2). The trials of all subjects were scored in randomized order. Before interpreting the study outcome, the intrarater and interrater reliabilities were determined. To obtain the intrarater reliability, each rater performed repeated measurements of all temporal, spatial, and visuoperceptual variables during the second swallow of each electrode position for all subjects (patients and healthy control subjects) within a period of 2 weeks.

\section{Statistical Analysis}

All temporal and spatial variables had six technical replications for each subject, as these were repeatedly measured in three different electrode positions (suprahyoidal, infrahyoidal, and bilateral combination) during two electrical current states (on/off), whereas the visuoperceptual variables had 12 technical measurements for each subject. Variables were measured for all electrode positions and electrical current states in both healthy control subjects and Parkinson's patients (Table 1). Random intercept models were fit for all reliable VFS outcome parameters. This was done twice: for the patients' only and for all subjects. In the former case, the objective was to determine whether the electrode positions and/or the status of the electrical current affected the VFS outcomes of Parkinson's patients. Patients were defined as random effects, whereas electrode positions and status of the electrical current were defined as fixed effects. This model accounts for the correlation induced by the nesting of repeated measurements within subjects. The alternative analyses, using all subjects, aimed at testing additionally for group differences, while concurrently adjusting for electrode position and electrical current status. However, for all subjects the random effect was no longer a single subject but a pair, composed of one patient and his/her matched healthy control (10 pairs in total). This minor change was necessary in order to accommodate dependence induced by matching. As in the case of patients only, the fixed effects consisted of electrode positions and electrical current states, in addition to the group (patient versus control). 
Table 2 Variables with good intrarater and interrater reliability [15]

\begin{tabular}{|c|c|c|}
\hline $\begin{array}{l}\text { Reliable }^{\mathrm{a}} \text { parameters in } \\
\text { videofluoroscopy }\end{array}$ & Name & Definition \\
\hline \multirow[t]{8}{*}{ Temporal parameters } & $\begin{array}{l}\text { VPJc (velopharyngeal junction } \\
\text { closure) }\end{array}$ & $\begin{array}{l}\text { Moment of first contact of the soft palate against the } \\
\text { posterior pharyngeal wall (in seconds) }\end{array}$ \\
\hline & $\begin{array}{l}\text { VPJo (velopharyngeal junction } \\
\text { opening) }\end{array}$ & $\begin{array}{l}\text { Moment of separation of the soft palate and the posterior } \\
\text { pharyngeal wall with re-entry of air in the retrolingual space from the } \\
\text { nasopharynx (in seconds) }\end{array}$ \\
\hline & $\begin{array}{l}\text { VPJd (velopharyngeal junction } \\
\text { duration) }\end{array}$ & $\Delta \mathrm{T}$ between VPJc and VPJo (in seconds) \\
\hline & LVc (laryngeal vestibule closure) & $\begin{array}{l}\text { Moment when laryngeal elevation results in making contact between } \\
\text { the arytenoid cartilages and the underside of the epiglottis (in seconds) }\end{array}$ \\
\hline & LVo (laryngeal vestibule opening) & $\begin{array}{l}\text { Moment of separation of the arytenoid cartilages and the underside of } \\
\text { the epiglottis with re-entry of air in the laryngeal vestibule (in seconds) }\end{array}$ \\
\hline & LVd (laryngeal vestibule duration) & $\Delta \mathrm{T}$ between $\mathrm{LVc}$ and $\mathrm{LVo}$ (in seconds) \\
\hline & $\begin{array}{l}\text { UESc (upper esophageal sphincter } \\
\text { closure) }\end{array}$ & Moment of closure of the esophagus after bolus transport (in seconds) \\
\hline & $\begin{array}{l}\text { GPJo (glossopalatal junction } \\
\text { opening) - LVc (laryngeal } \\
\text { vestibule closure) }\end{array}$ & $\Delta \mathrm{T}$ between GPJo and LVc (in seconds) \\
\hline \multirow{3}{*}{$\begin{array}{l}\text { Spatial and temporal } \\
\text { parameters of } \\
\text { hyoid motion }\end{array}$} & Vertical hyoid motion & Maximum vertical motion during swallowing act (in $\mathrm{mm}$ ) \\
\hline & Duration horizontal hyoid motion & $\begin{array}{l}\text { Duration between initiation of swallow and moment of maximum } \\
\text { horizontal (anterior) motion (in seconds) }\end{array}$ \\
\hline & Duration vertical hyoid motion & $\begin{array}{l}\text { Duration between initiation of swallow and moment of maximum vertical } \\
\text { motion (in seconds) }\end{array}$ \\
\hline \multirow{3}{*}{$\begin{array}{l}\text { Visuoperceptual } \\
\text { parameters }\end{array}$} & Lingual pumping & Preswallow involuntary repetitive tongue movements (5-point scale, $0-4$ ) \\
\hline & Piecemeal deglutition & Sequential swallowing of the same bolus (5-point scale, $0-4$ ) \\
\hline & Postswallow vallecular pooling & Postswallow pooling in the valleculae (3-point scale, $0-2$ ) \\
\hline
\end{tabular}

a Cronbach's alpha $>0.65$ or Cohen's kappa index of agreement $>0.60$, ICC $>0.60$

b Lower scores refer to normal functioning and higher scores refer to more severe disability

Model parameters were estimated via the restricted maximum likelihood method (REML), and parameters were considered statistically significant if the $P$ value of their corresponding likelihood ratio (LR) tests was smaller than 0.05. All statistical analyses were performed using SPSS version 16.0 software (SPSS Inc., Chicago, IL, USA).

\section{Results}

\section{Demographics}

Ten mentally competent dysphagic patients ( 3 women, 7 men) with a diagnosis of idiopathic Parkinson's disease and ten healthy control subjects matched for age and gender were included. The mean age of the patients and the healthy subjects was 66-65 years, respectively, with a maximum age difference of 4 years between patient and matched control. The $\mathrm{H} \& \mathrm{Y}$ scale ranged from mild to moderate $($ median $=$ II). The demographics of the patients and the healthy control subjects are presented in Table 1 . The patients had their Parkinson's disease from 5 to 13 years.

\section{Reliable Variables}

In Table 2, all variables with good intrarater and interrater reliabilities are presented [Cronbach's $\alpha>0.65$, Cohen's $\kappa$ index of agreement $>0.60$, and intraclass correlation coefficients (ICC) >0.60] [15]. ICCs and Cronbach's $\alpha$ were computed for all continuous variables. For all ordinal variables, Cohen's $\kappa$ index of agreement was used. Ratings on visuoperceptual ordinal variables like preswallow anterior and preswallow posterior spill, swallow hesitancy, and postswallow oral residue showed no abnormalities. It was decided to exclude these variables because of insufficient relevance to the present Parkinson patient group. The intrarater reliability was not sufficient for the variables delayed initiation pharyngeal reflex, postswallow pyriform sinus pooling, the penetration-aspiration scale, GPJo, GPJc, and UESo. When the intrarater reliability proved to be rather 
low (Cohen's $\kappa$ index of agreement $<0.60$, ICC $<0.60$ ), the interrater reliability was not computed.

\section{Effect of Electrical Stimulation}

Statistical tests for electrical stimulation and/or electrode position did not reveal statistical significance for the vast majority of the VFS parameters in the patient stratum, except for the temporal variable laryngeal vestibule duration (LVd) (Fig. 3) and the spatiotemporal variable duration horizontal hyoid motion (Fig. 4). In LVd, time on average was significantly reduced in position II (electrodes are placed horizontally below the hyoid bone) compared to position III (combination of position I and II), whereas in duration horizontal hyoid motion, time was significantly increased in position II compared to position I (electrodes are placed horizontally above the hyoid bone) (Table 3 ). This same effect was observed for the control group (Fig. 4). The general lack of significance for electrode positions and electrical current status was strongly confirmed by the random intercept model using all data (patients and healthy control subjects), which showed that these factors did not rise above individual differences. By contrast, once adjusted for electrode position and electrical current status in the statistical model, significant group differences emerged (Table 4). In Fig. 5, for instance, box plots of the temporal variable UESc of the individual matched pairs are displayed. The objective of this illustration is twofold. First, it allows for visualization of all

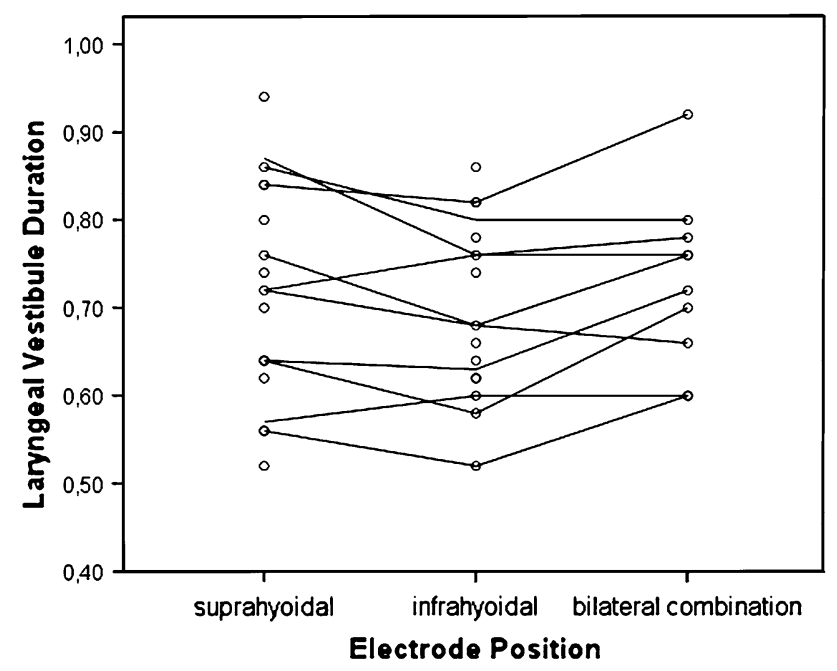

Fig. 3 Observed mean LVd (laryngeal vestibule duration) values (in seconds) for each patient (individual lines) per electrode position, averaged over the two electrical current states (current on vs. off). The dots represent the electrical current status (current on/off) per patient and per electrode position. The electrode position as a fixed effect, which can be thought of as an average over patients' individual lines, was shown to be significantly reduced in position II compared to position III

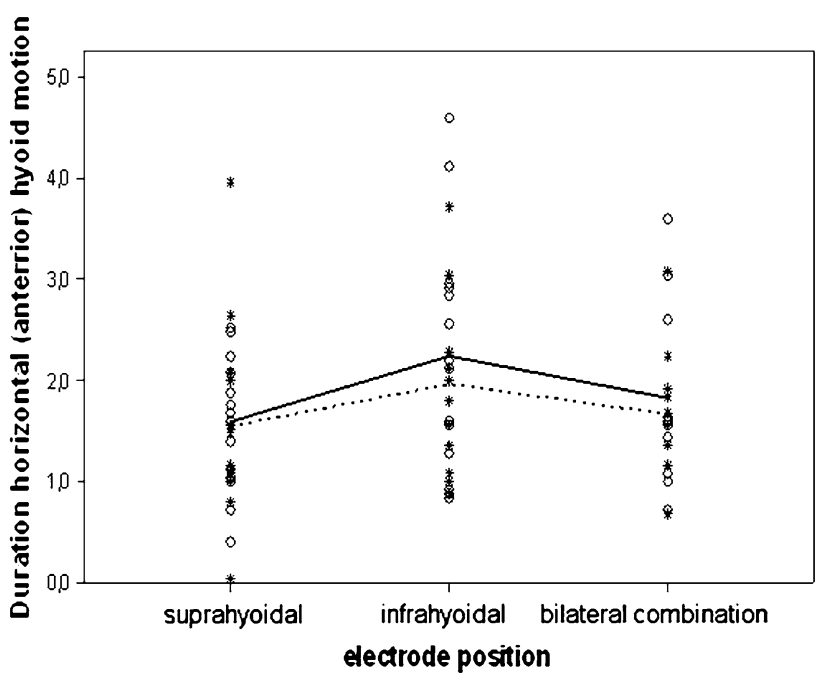

Fig. 4 Observed values of duration horizontal (anterior) hyoid motion (in seconds), marked for both groups separately (open circle $=$ patients and asterisk $=$ controls, solid and dotted lines, respectively) (all data analysis). Note the marginally higher values for the infrahyoidal position (II) for the two groups, detected as statistically significant regardless of the electrical current status (current on/off). Differences between patients and controls were nonsignificant

data (patients and healthy control subjects), highlighting the nesting of the subjects within pairs. Second, it shows that for six of the ten patient-control pairs, the patients had values that are larger than or comparable to those for the controls, resulting in the statistically significant difference observed between the two groups. The significant results of the random intercept models using patient data and all data (patients and healthy control subjects) are summarized in Tables 3 and 4, where the marginal estimated mean values for the significant fixed effects are given (the current on/off effect is omitted due to its nonsignificance).

\section{Discussion}

Studying therapy effects for dysphagia in Parkinson's disease using surface electrical stimulation is a challenge. In previous studies, several authors have investigated the effects of surface electrical stimulation in other populations such as chronically dysphagic patients, stroke patients, or healthy subjects $[3,4,16]$. However, the pathological mechanisms of oropharyngeal dysphagia in Parkinson patients are quite different from the mechanisms of chronic dysphagia in many other diseases, e.g., dysphagia following treatment for head and neck cancer or stroke. The pathophysiology of dysphagia in Parkinson's disease is complex and dependent on the coordination among both peripheral and central nervous system factors; uncoordinated or disrupted signals along these pathways 
Table 3 Random intercept models using patient data

\begin{tabular}{|c|c|c|c|}
\hline $\begin{array}{l}\text { VFS } \\
\text { parameters }\end{array}$ & $\begin{array}{l}\text { Electrode } \\
\text { position }^{\mathrm{a}}\end{array}$ & $\begin{array}{l}\text { Comparison between } \\
\text { electrode positions }{ }^{\mathrm{b}}\end{array}$ & \\
\hline \multirow{4}{*}{$\begin{array}{l}\text { Laryngeal } \\
\text { vestibule } \\
\text { duration }^{\mathrm{c}} \\
\text { (seconds) }\end{array}$} & Position I: & Position I versus II: & NS \\
\hline & $0.72(0.03)$ & $0.03(-0.013,0.07)$ & \\
\hline & $\begin{array}{l}\text { Position II: } \\
0.69(0.03)\end{array}$ & $\begin{array}{l}\text { Position II versus III: } \\
-0.05(-0.097,-0.003)\end{array}$ & Sign. ${ }^{c}$ \\
\hline & $\begin{array}{l}\text { Position III: } \\
0.74(0.03)\end{array}$ & $\begin{array}{l}\text { Position III versus I: } \\
0.022(-0.025,0.069)\end{array}$ & NS \\
\hline \multirow{3}{*}{$\begin{array}{l}\text { Duration } \\
\text { horizontal } \\
\text { hyoid } \\
\text { motion }^{\mathrm{d}} \\
\text { (seconds) }\end{array}$} & $\begin{array}{l}\text { Position I: } \\
1.54(0.27)\end{array}$ & $\begin{array}{l}\text { Position I versus II: } \\
-0.737(-1.288,-0.186)\end{array}$ & Sign. ${ }^{d}$ \\
\hline & $\begin{array}{l}\text { Position II: } \\
2.27(0.28)\end{array}$ & $\begin{array}{l}\text { Position II versus III: } \\
0.488(-0.139,1.116)\end{array}$ & NS \\
\hline & $\begin{array}{l}\text { Position III: } \\
1.78(0.33)\end{array}$ & $\begin{array}{l}\text { Position III versus I: } \\
0.248(-0.379,0.876)\end{array}$ & NS \\
\hline
\end{tabular}

Significant VFS parameters and the estimated means plus standard errors per electrode position and the mean differences between electrode positions in combination with the $95 \%$ confidence intervals (according to the fitted random intercept models). These VFS parameters show significant differences for electrode position regardless of the electrical current status (current on/off)

a Values are mean (standard error)

b Values are mean difference $(95 \% \mathrm{CI})$

c Statistically significant pairwise difference between II and III

d Statistically significant pairwise difference between I and II

(dopaminergic and nondopaminergic) can lead to oropharyngeal dysphagia. Several investigators have designed therapy-effect studies based on treatment of dysphagic features that are considered specific to Parkinson's disease, including rigidity, hypokinesia, and bradykinesia of the oral or pharyngeal stages of swallowing, incomplete cricopharyngeal relaxation, reduced cricopharyngeal opening, and delayed initiation of the swallowing reflex [17-19]. Therapies aimed at reduction of the aspiration risk in Parkinson's disease can include voluntary airway protection techniques and bolus modification [20-22].

In the present study, differences in swallowing physiology during a single-session protocol of surface electrical stimulation were investigated in Parkinson patients and age- and gender-matched healthy control subjects using qualitative and quantitative variables in videofluoroscopy that have proved to be sufficiently reliable (Table 2).

Poor reliability was observed for the penetration-aspiration scale, among others variables. Diverse situations may have contributed to this finding. First, despite their high level of expertise, the strict methodological protocol for repeated measurements, and the consensus training, the raters may have lacked consensus on a definition of the levels of the scale. Second, patients were found to be rather homogeneous with respect to several of the variables being measured, i.e., some variables such as preswallow anterior spill, preswallow posterior spill, and the penetration-aspiration scale almost always scored consistently the same level of the ordinal scale.

For most temporal, spatial, and visuoperceptual variables, no statistically significant changes were found during surface electrical stimulation. For the temporal variable laryngeal vestibule duration (LVd), a significant effect was found for position II (in which the electrodes are placed horizontally below the hyoid bone) compared to position III (combination of positions I and II) regardless of the status of the electrical current in the Parkinson and healthy control groups, possibly indicating a placebo effect. The value of this temporal measurement is significantly reduced in position II compared to position III, resulting in a decreased period of laryngeal closure. Although the risk of

Table 4 Random intercept models using all data (patients and healthy control subjects)

\begin{tabular}{llrl}
\hline VFS parameters & Group & $\begin{array}{l}\text { Mean } \\
\text { (standard error) }\end{array}$ & $\begin{array}{l}\text { Mean difference } \\
\text { between patients } \\
\text { and controls (95\% CI) }\end{array}$ \\
\hline Velopharyngeal junction closure (seconds) & Patients & $0.054(0.03)$ & $0.059(0.002,0.117)$ \\
Laryngeal vestibule closure (seconds) & Controls & $-0.005(0.03)$ & $0.152(0.024,0.277)$ \\
Laryngeal vestibule duration (seconds) & Controls & $0.16(0.06)$ & $-0.32(-0.642,-0.003)$ \\
Upper esophageal sphincter closure (seconds) & Patients & $0.008(0.06)$ & $0.73(0.05)$ \\
Controls & Patients & $1.05(0.05)$ & $0.07(0.001,0.127)$ \\
Piecemeal deglutition (5-point scale, 0-4) & Controls & $0.87(0.04)$ & $0.80(0.04)$ \\
& Patients & $1.248(0.17)$ & $0.557(0.235,0.878)$
\end{tabular}

Significant VFS parameters and their estimated means plus standard errors for patients and controls separately, in combination with the $95 \% \mathrm{CI}$ of the mean difference between both groups. In the random intercept model, the data have been adjusted for electrode position and electrical current status (current on/off) which are both not significant 


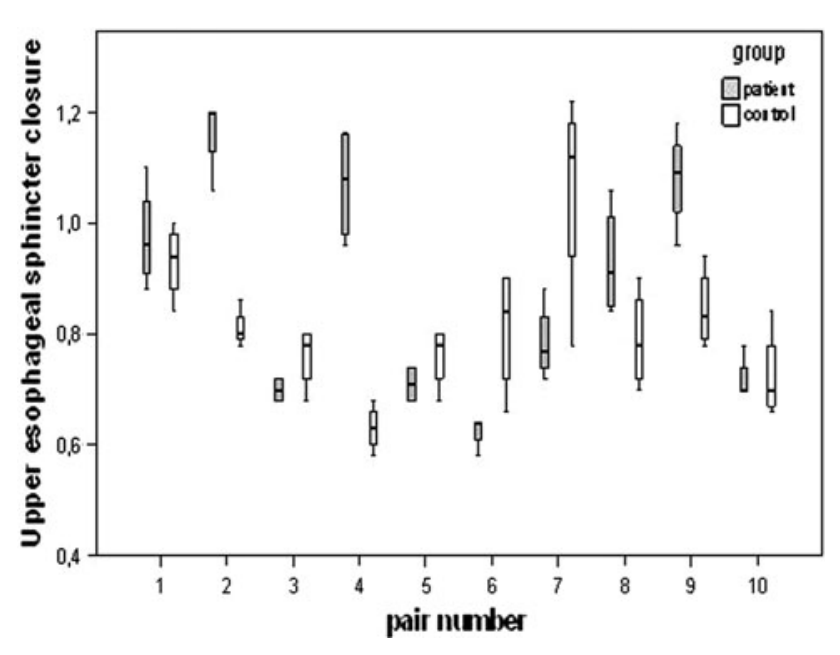

Fig. 5 Box plots of UESc (upper esophageal sphincter closure in seconds) showing the nesting of subjects within each matched patient-control pair. For UESc a statistically significant difference is observed between the two groups after adjustment for electrode position and electrical current status (current on/off) which are both not significant

penetration or even aspiration may not be increased in the present Parkinson population, it may be increased in patients with more severe Parkinson disease due to a decreased period of laryngeal closure. For the spatially related temporal variable duration horizontal hyoid motion, a significant effect is found for electrode position II compared to position I (electrodes are placed horizontally above the hyoid bone), again regardless of the status of the electrical current in Parkinson patients and healthy control subjects. The value of this variable increases significantly in position II compared to position I, indicative of the hyoid motion being slowed down.

It is known that several therapies induce a placebo effect in Parkinson patients and in healthy volunteers [23, 24]. The presence of skin electrodes and the connection of the electrical stimulation device may have worked as a sham intervention. The lack of a significant effect of surface electrical stimulation in the present study may not be a simple issue of statistical power. Continuous and ordinal variables were assessed per swallow $(N=240$ swallows in total), resulting in 4800 measurements. It was expected that this number of swallows and variables would reveal significant differences or at least indicate possible tendencies in measurement data.

The fixed stimulation variables (frequency $=80 \mathrm{~Hz}$, pulse width $=700 \mu \mathrm{s}$, current intensity $=0-25 \mathrm{~mA}$ ) of the VitalStim electrical stimulator may not have been optimal for inducing any effects during the applied singlesession protocol of surface electrical stimulation in Parkinson's patients. Oropharyngeal excitability depends on the stimulation variables [25]. Therefore, different values of the stimulation variables may cause different effects in
Parkinson's disease. Oropharyngeal dysphagia in the presence of Parkinson's disease can be due to dysfunction of central nervous system pathways of swallowing rather than muscle weakness or peripheral sensory dysfunction for which the applied electrical stimulation device was mainly designed for $[26,27]$. The possibility that snap skin electrodes may not be a selective technique of neuromuscular stimulation cannot be disregarded too. It is likely that muscles or other tissues not involved in swallowing physiology or antagonistic muscles (muscles that pull the hyoid bone upward or downward) have been stimulated simultaneously, thereby not resulting in a measurable effect in VFS [4]. Synchronous electromyographic (EMG) feedback of the supra- and infrahyoidal muscles was not performed to exclude this phenomenon. Intramuscular stimulation as described by Burnett et al. [28] provides a more precise technique of electrical stimulation, although many patients may not be willing to accept invasive electrodes. In addition to these explanations, the lack of significant effects may also be caused by the choice of the assessment tools as well as the outcome variables. VFS may not be the most optimal assessment tool to indicate possible effects of surface electrical stimulation in dysphagic Parkinson patients. Ertekin et al. [26] described conflicting results of radiological studies. Some of those studies did not detect any abnormalities in the swallowing of Parkinson patients, unlike other studies that found abnormalities in similar populations of Parkinson patients [26]. Furthermore, normal motility of the UES region during VFS was observed despite the presence of manometric abnormalities in the same region [26, 29]. Other assessment tools such as EMG or manometry may be more sensitive and reveal significant effects [26]. However, as the assessment protocol was already a burden on the patients, it was decided not to subject them to additional assessment tools.

Another reason for finding few significant results from the use of surface electrical stimulation can be the relatively intact swallow physiology in the early phase of Parkinson's disease as observed in this pilot population. The population of included patients is a realistic representation of Parkinson patients who consult their speech therapists about dysphagic complaints. For several mainly logistical reasons (e.g., not being able to sit upright for VFS, condition too weak for repeated transport to the outpatient clinic for dysphagia, and suffering from Parkinson dementia), patients with severe Parkinson's disease, who are often admitted to nursing homes, did not find easy access to this study. On the other hand, following adjustment for electrode position and electrical current status in the random intercept models (Table 4), several variables show significant group differences (patients versus healthy control group) despite the early phase of Parkinson's 
disease. These group differences may be a result of pathophysiological changes in swallowing due to Parkinson's disease after all. Several temporal variables show significant differences: moment of velopharyngeal junction closure, moment of laryngeal vestibule closure, and moment of upper esophageal sphincter closure appear significantly later in the swallow of Parkinson patients compared to that of healthy control subjects. As stated earlier, rigidity, hypokinesia, and bradykinesia of the oral or pharyngeal stage of swallowing in Parkinson's disease may be the reason for these significant differences [17-19]. The duration of the laryngeal vestibule closure (laryngeal vestibule duration) is significantly shorter in Parkinson patients compared to healthy control subjects. This phenomenon may result in a less protected airway in Parkinson patients [30]. The visuoperceptual variable piecemeal deglutition (sequential swallowing of the same bolus) has a significant higher score in Parkinson patients compared to healthy control subjects. Parkinson patients need more swallows for the same liquid bolus before the entire bolus has entered the esophagus. Possible explanations are oropharyngeal muscle weakness, peripheral sensory dysfunction, or central nervous system dysfunction in Parkinson's disease resulting in less efficient bolus formation, delayed oropharyngeal bolus transition, and aspiration $[26,31]$.

\section{Conclusion}

A single session of surface electrical stimulation using different electrode positions in dysphagic Parkinson patients during VFS resulted in only a few statistically significant effects. The present study provides preliminary data which shows that surface electrical stimulation can alter swallowing in Parkinson's disease. However, these effects may have been caused mainly by the placebo effect. The changes measured from surface electrical stimulation were found in both healthy control subjects and Parkinson patients and the direction of change would not likely benefit swallowing. Questions arise about the mechanism of surface electrical stimulation and its effects on the neural pathways involved in swallowing. Following adjustment in the statistical model for electrical current status as well as electrode positions (both not significant), additional information appeared on group differences between Parkinson patients and healthy control subjects for several variables.

Further studies using, for example, larger patient populations, a sham intervention group, different electrical stimulation variables (frequency, amplitude, and pulse width), or EMG feedback are necessary to evaluate the potential therapeutic effect of electrical stimulation in dysphagic patients with Parkinson's disease. Furthermore, as stated in an earlier study by Baijens et al. [15], the intrarater and interrater reliabilities in outcome studies such as this one and in future research remains a major concern. In order to determine the effects of therapy interventions on swallowing and to compare study outcomes for different subject populations, measurement protocols must be standardized and based on reproducible, reliable ratings of well-defined variables.

Disclosures The authors declare that they have no conflict of interest.

Open Access This article is distributed under the terms of the Creative Commons Attribution Noncommercial License which permits any noncommercial use, distribution, and reproduction in any medium, provided the original author(s) and source are credited.

\section{References}

1. Baijens LWJ, Speyer R. Effects of therapy for dysphagia in Parkinson's disease. Systematic review. Dysphagia. 2009;24:91102.

2. Carnaby-Mann GD, Crary MA. Examining the evidence on neuromuscular electrical stimulation for swallowing: a metaanalysis. Arch Otolaryngol Head Neck Surg. 2007;133:564-71.

3. Humbert I, Poletto C, Saxon K, Kearney PR, Crujido L, WrightHarp W, Payne J, Jeffries N, Sonies B, Ludlow CL. The effect of surface electrical stimulation on hyo-laryngeal movement in normal individuals at rest and during swallowing. J Appl Physiol. 2006;101:1657-63.

4. Ludlow CL, Humbert I, Saxon K, Poletto C, Sonies B, Crujido L. Effects of surface electrical stimulation both at rest and during swallowing in chronic pharyngeal dysphagia. Dysphagia. 2007; 22:1-10.

5. Clark H, Lazarus C, Arvedson J, Schooling T, Frymark T. Evidence-based systematic review: effects of neuromuscular electrical stimulation on swallowing and neural activation. Am J Speech Lang Pathol. 2009;18:361-75.

6. Hoehn NM, Yahr MD. Parkinsonism: onset, progression and mortality. Neurology. 1967;17:427-42.

7. Folstein MF, Folstein SE, McHugh PR. Mini-mental state. A practical method for grading the cognitive state of patients for the clinician. J Psychiatr Res. 1975;12:189-98.

8. Wajsbort J. The "off-on" phenomenon during treatment of Parkinson's disease with levodopa. J Neurol. 1977;215:59-66.

9. Clavé P, de Kraa M, Arreola V, Girvent M, Farré R, Palomera E, Serra-Prat M. The effect of bolus viscosity on swallowing function in neurogenic dysphagia. Aliment Pharmacol Ther. 2006;24:1385-94.

10. Kahrilas PJ, Lin S, Rademaker A, Logemann J. Impaired deglutitive airway protection: a videofluoroscopic analysis of severity and mechanism. Gastroenterology. 1997;113:1457-64.

11. Rosenbek JC, Robbins JA, Roecker EB, Coyle JL, Wood JL. A penetration-aspiration scale. Dysphagia. 1996;11:93-8.

12. Logemann J, Kahrilas PJ, Begelman J, Dodds WJ, Pauloski BR. Interactive computer program for biomechanical analysis of videoradiographic studies of swallowing. AJR Am J Roentgenol. 1989;153:277-80.

13. Robbins JA, Logemann J, Kirshner H. Swallowing and speech production in Parkinson's disease. Ann Neurol. 1986;19:283-7.

14. Nagaya M, Teruhiko K, Yamada T, Igata A. Videofluorographic study of swallowing in Parkinson's disease. Dysphagia. 1998;13: 95-100. 
15. Baijens LW, Speyer R, Passos VL, Pilz W, Roodenburg N, Clavé P. Swallowing in Parkinson patients versus healthy controls: Reliability of measurements in videofluoroscopy. Gastroenterol Res Pract. 2011;2011:380682.

16. Bülow M, Speyer R, Baijens L, Woisard V, Ekberg O. Neuromuscular electrical stimulation (NMES) in stroke patients with oral and pharyngeal dysfunction. Dysphagia. 2008;23:302-9.

17. Pinnington LL, Muhiddin KA, Ellis RE, Playford ED. Noninvasive assessment of swallowing and respiration in Parkinson's disease. J Neurol. 2000;47:773-7.

18. Lim A, Leow L, Huckabee ML, Frampton C, Anderson T. A pilot study of respiration and swallowing integration in Parkinson's disease: "on" and "off" levodopa. Dysphagia. 2008;23:76-81.

19. Nagaya M, Kachi T, Yamada T. Effect of swallowing training on swallowing disorders in Parkinson's disease. Scand J Rehabil Med. 2000;32:11-5.

20. Bushmann M, Dobmeyer SM, Leeker L, Perlmutter JS. Swallowing abnormalities and their response to treatment in Parkinson's disease. Neurology. 1989;39:1309-14.

21. Troche MS, Sapienza CM, Rosenbek JC. Effects of bolus consistency on timing and safety of swallow in patients with Parkinson's disease. Dysphagia. 2008;23:26-32.

22. Logemann JA, Gensler G, Robbins J, Lindblad AS, Brandt D, Hind JA, Kosek S, Dikeman K, Kazanjian M, Gramigna GD, Lundy D, McGarvey-Toler S, Miller Gardner PJ. A randomized study of three interventions for aspiration of thin liquids in patients with dementia or Parkinson's disease. J Speech Lang Hear Res. 2008;51:173-83.

23. Pollo A, Benedetti F. The placebo response: neurobiological and clinical issues of neurological relevance. Prog Brain Res. 2009;175:283-94.

24. Rosenzweig P, Brohier S, Zipfel A. The placebo effect in healthy volunteers: influence of experimental conditions on the adverse events profile during phase I studies. Clin Pharmacol Ther. 1993;54:578-83.

25. Fraser C, Rothwell J, Power M, Hobson A, Thompson D, Hamdy S. Differential changes in human pharyngoesophageal motor excitability induced by swallowing, pharyngeal stimulation, and anesthesia. Am J Physiol Gastrointest Liver Physiol. 2003;285: G137-44.

26. Ertekin C, Tarlaci S, Aydogdu I, Kiylioglu N, Yuceyar N, Turman AB, Secil Y, Esmeli F. Electrophysiological evaluation of pharyngeal phase of swallowing in patients with Parkinson's disease. Mov Disord. 2002;17:942-9.

27. Freed M, Wijting Y. VitalStim certification program. Training manual for patient assessment and treatment using VitalStim electrical stimulation. Hixson: Chattanooga Group; 2003.

28. Burnett TA, Mann EA, Cornell SA, Ludlow CL. Laryngeal elevation achieved by neuromuscular stimulation at rest. J Appl Physiol. 2003;94:128-34.

29. Ali GN, Wallace KL, Schwartz R, DeCarle DJ, Zagami AS, Cook IJ. Mechanisms of oral-pharyngeal dysphagia in patients with Parkinson's disease. Gastroenterology. 1996;110:383-92.

30. Kendall KA, Leonard RJ. Bolus transit and airway protection coordination in older dysphagic patients. Laryngoscope. 2001; 111:2017-21.

31. Troche MS, Okun MS, Rosenbek JC, Musson N, Fernandez HH, Rodriguez R, Romrell J, Pitts T, Wheeler-Hegland KM, Sapienza CM. Aspiration and swallowing in Parkinson disease and rehabilitation with EMST: a randomized trial. Neurology. 2010;23: 1912-9.

Laura W. J. Baijens MD

Renée Speyer $\mathrm{PhD}, \mathrm{MSc}$, SLP

Valeria Lima Passos $\mathrm{PhD}$, MPhil

Walmari Pilz SLP

Nel Roodenburg SLP

Père Clavé $\mathrm{MD}, \mathrm{PhD}$ 